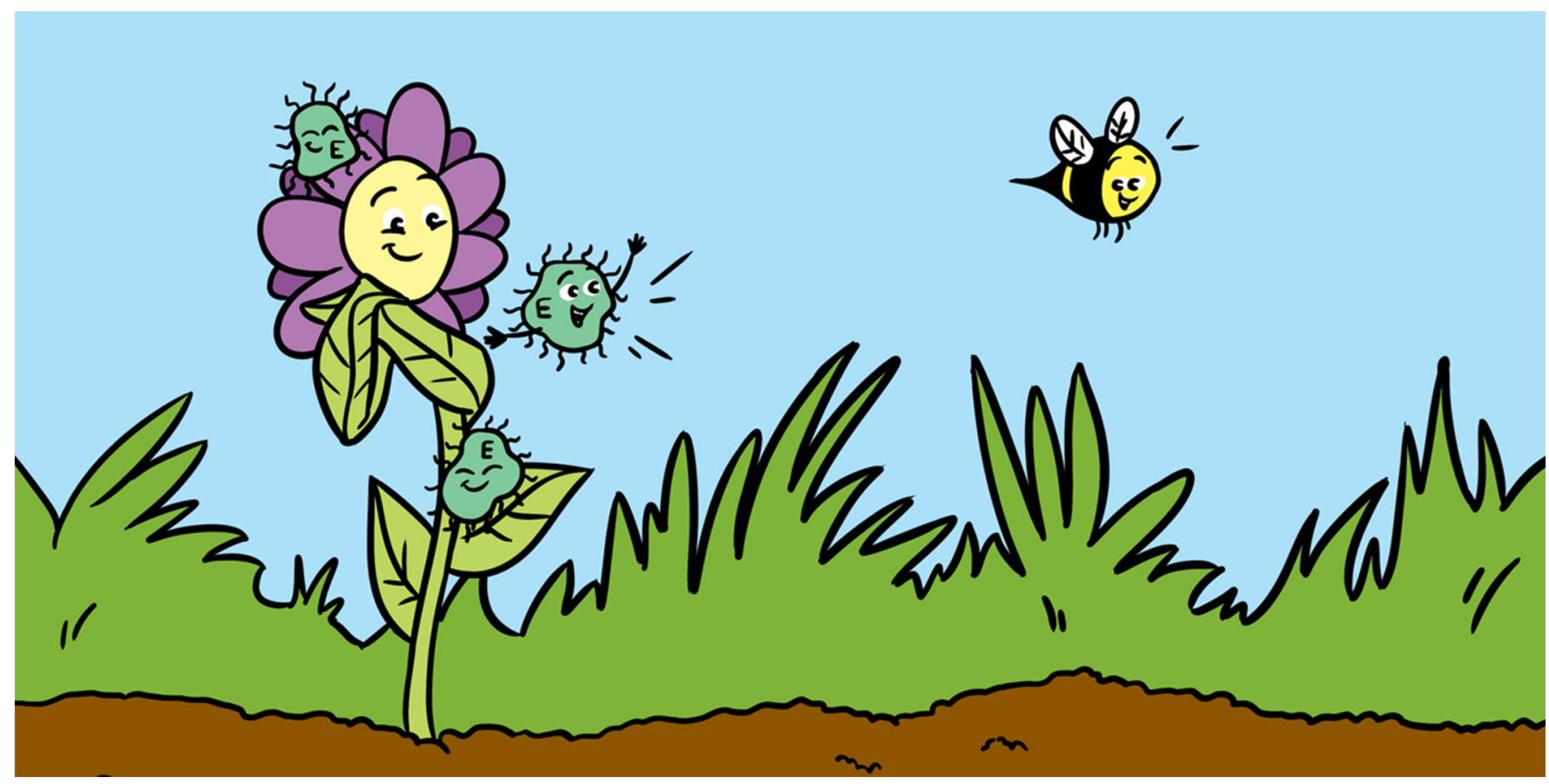

\title{
HIDDEN MICROBIAL HELPERS LIVING INSIDE PLANTS: GETTING TO KNOW ENDOPHYTES AND SOME OF THEIR APPLICATIONS IN OUR DAILY LIVES
}

\section{Rosario del Carmen Flores-Vallejo ${ }^{1 *}$, Jorge Luis Folch-Mallol ${ }^{2}$, Ashutosh Sharma ${ }^{3}$, Alexandre Toshirrico Cardoso-Taketa ${ }^{1}$, María Luisa Villarreal ${ }^{1 *}$}

\footnotetext{
Laboratorio de Investigación de Plantas Medicinales, Centro de Investigación en Biotecnología, Universidad Autónoma del Estado de Morelos, Cuernavaca, Mexico

2 Laboratorio de Biología Molecular de Hongos, Centro de Investigación en Biotecnología, Universidad Autónoma del Estado de Morelos, Cuernavaca, Mexico

Escuela de Ingeniería y Ciencias, Tecnológico de Monterrey, Santiago de Querétaro, Mexico
}

\section{YOUNG REVIEWERS:}



ALAN

AGE: 13

MARIANA

AGE: 14
Microbes are tiny forms of life that can be found everywhere. The microbes that live inside healthy plants are commonly known as endophytes. Plants live together with their endophytes in symbiosis, which means that they work together to help each other out. Plants give shelter and food to their microbial guests, and in exchange, their microbial helpers produce chemicals that support the plants to grow faster, resist droughts, or fight against plant eaters. Scientists are very interested in studying the chemicals produced by endophytes, because new medicines and agricultural products can be developed from them. In this article, we will describe the interactions between plants and their endophytes, and answer some questions, like: How do scientists 
select a plant host and extract endophytes from plants? How could the study of endophytes help us in our daily lives? And, which types of endophytes have been isolated from plants growing in Mexico?

\section{PLANTS AND THEIR HIDDEN MICROBIAL HELPERS: ENDOPHYTES}

Plants are living organisms that, unlike other forms of life, cannot move to escape from animals or insects that want to eat them (herbivores). So, how did plants compensate for their lack of movement and continue to survive? Well, since very ancient times, plants evolved together with microbes, which are tiny organisms, invisible to the naked eye. This cooperation with microbes is one of the strategies that helped plants to adapt, survive, and thrive [1]. Microbes that live inside the healthy tissues of plants, without causing disease are commonly known as endophytes (from the Latin words "endo" = inside, and "phyton" = plant).

Endophytes include microbes like fungi, bacteria, and even viruses.

\section{SYMBIOSIS}

\section{A long-term} relationship in which organisms benefit from each other.

\section{Figure 1}

Plants and their endophytes work together in symbiosis (A) Endophytes living inside the tissues of their plant host, where the plant gives them shelter and nutrients. (B) An example of how endophytes help to protect their plants. (1) A herbivore attacks the plant; (2) the plant sends signals that warn its endophytes about the attack;

(3) endophytes respond to the plant's signals and help to produce chemicals; (4) the chemicals are secreted by the plant and reach the herbivore; and (5) the herbivore gets repelled by the chemicals produced with the help of the endophytes.
Endophytes and their plant hosts work together in symbiosis, which means they both obtain benefits from their relationship. When working in symbiosis, plants send signals to their endophytes to warn them when they are being attacked by herbivores. In response, endophytes help to produce chemicals that their plant hosts use to stop herbivores from eating the plant. Endophytes can also help to produce other types of useful chemicals (Figure 1). For example, chemicals that: (1) attract pollinators, like bees and bats; (2) help their plant host to grow faster and absorb the nutrients in the soil more easily; (3) make their plant host resistant to drought and extreme weather conditions; and (4) fight plant infections and diseases. In exchange for those services, plants give shelter and food to their endophytes, which helps the endophytes

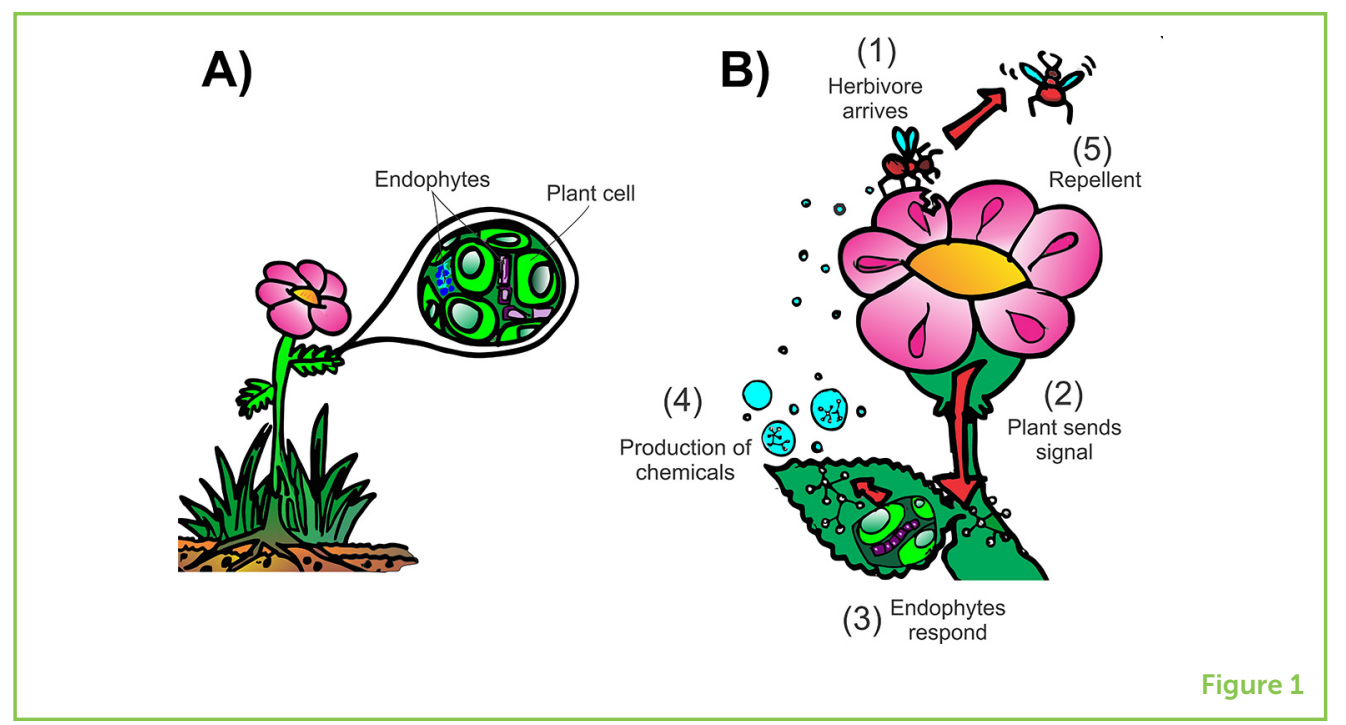




\section{EPIPHYTES}

Microbes living in the surface of plants.

PHYTOPATHOGENS

Microbes that cause disease to plants

\section{BIOTECHNOLOGIES}

Products and services developed from living organisms or their parts. to reproduce and gives them a chance to be passed on to new plants through the seeds of their plant host [1].

At this point, you might be wondering how endophytes get inside plants. Plants have an immune system that normally defends them from anything that tries to invade their cells, including the microbes that are normally found on the plant's outer surface, known as epiphytes. Under certain conditions, when the plant's immune system is weakened, epiphytes take advantage and enter the plant through wounds on the roots or the leaves. Inside the plant, some of these microbes will manage to co-exist with the plant's immune system without causing any symptoms of disease - these become endophytes-while some others will provoke sickness to the plant, which are known as phytopathogens [1].

\section{HOW COULD ENDOPHYTES HELP US IN OUR DAILY LIVES?}

Scientists started paying more attention to the study of endophytes when one group demonstrated that endophytes could produce chemicals that were originally thought to be produced by plants. This group discovered an endophyte capable of producing a chemical named Paclitaxel $\left(\operatorname{Taxol}^{\circledR}\right)$, which is used in the treatment of cancer. This chemical is mainly obtained from bark of the Pacific yew tree (Taxus brevifolia). To extract enough of this chemical and treat patients with ovarian and breast cancer for 1 year, it is estimated that an average of 270,000 mature trees (100 years old) must be chopped down, endangering the already reduced populations of this tree [2]. Their discovery that endophytes can produce Paclitaxel means that we might have a feasible way to produce it using microorganisms, without endangering the survival of the Pacific yew tree. This finding also encouraged scientists to explore the microbes that live inside plants, to "hunt" for yet-unknown species capable of producing valuable chemicals that could be useful to humans.

Studying endophytes might bring us a variety of new biotechnologies that could help us in our daily lives. For instance: (1) development of new medicines and ways to manufacture them; (2) creation of products that resist extreme temperatures or corrosive conditions; (3) discovery of new ways to decontaminate polluted areas; (4) creation of products that allow crops to be grown with less fertilizer and water; and (5) discovery of environmentally friendly ways of fighting unwanted herbivores or plant diseases in a more sustainable way. 


\section{ON THE LOOKOUT FOR ENDOPHYTES}

Endophytes live inside all plant species. They have been found in plants living on the sea, like green algae and plants that live on land, such as the dandelions growing near the sidewalk. Endophytes can live in plants from places with high temperatures and little water, like cacti, or inside tropical palm trees and mangroves from the coastal shores, or in ferns and mosses that live in the cold Antarctic zone [3]. If endophytes live in every plant, how do we choose which plant to study for its endophytes?

While it is true that every plant contains at least one relevant endophyte, the relevance of an endophyte relies on the function that it carries out on the plant and the benefits that the endophyte provides (e.g., help its plant host to resist drought). Some endophytes develop a very close association with their plant host, and contribute to different processes crucial for the survival of the plant. In order to study the biological activity that endophytes carry on inside their plant, first we must select a plant specimen.

There are different ways to select plants to study their endophytes. The selection of a plant is guided by different criteria that go from random selection of a number of different plants in an ecosystem, hoping to find something useful. Or the selection might be guided by certain characteristics of the plant to find chemicals with interesting biological activity (bioprospecting). This rationale of selection is based on: the plant's particular morphology, age, ecological niche, environmental setting, and/or the ethnobotanical history (medicinal uses) [3]. By applying rationales of selection to choose a particular plant and study its endophytes researchers can propose relevant experiments to test their research question (e.g., test if endophytes from desert plants produce chemicals that help their plant host to resist dehydration). The research question is what guides the whole scientific process of discovery, and helps scientists to explain the functions that endophytes carry inside their plant host, and understand their ability to produce chemicals with a particular biological activity.

There are different rationales of selection that guide scientists to choose one particular plant and perform bioprospecting from endophytes [3]. For instance, the scientists that discovered the Paclitaxel-producing endophyte followed an ethnomedicinal rationale, and selected a plant that had known medicinal uses. In their case, the plant was known to be used for treating cancer-like symptoms (owed to the presence Paclitaxel in its bark). Other scientists might use an ecologic rationale, by selecting plants that remain healthy even when nearby plants have diseases or are being attacked by herbivores. These plants might have endophytes that can produce chemicals that can ward off insects or bacteria. Another way to choose plants to study is by an adaptability 
Figure 2

Bioprospecting for endophytes. Rationales followed to select a specific plant to find endophytes with interesting biological activities:

(1) Ethnomedicinal: plants with records of medicinal uses;

(2) Ecologic: plants that resist diseases and herbivore's attack;

(3) Ecosystemic: plants found on habitats with high biodiversity;

(4) Endemic:

indigenous and

long-lived plants from a particular region;

(5) Morphologic: plants with uncommon physical features;

(6) Adaptability: plants that thrive in extreme conditions.

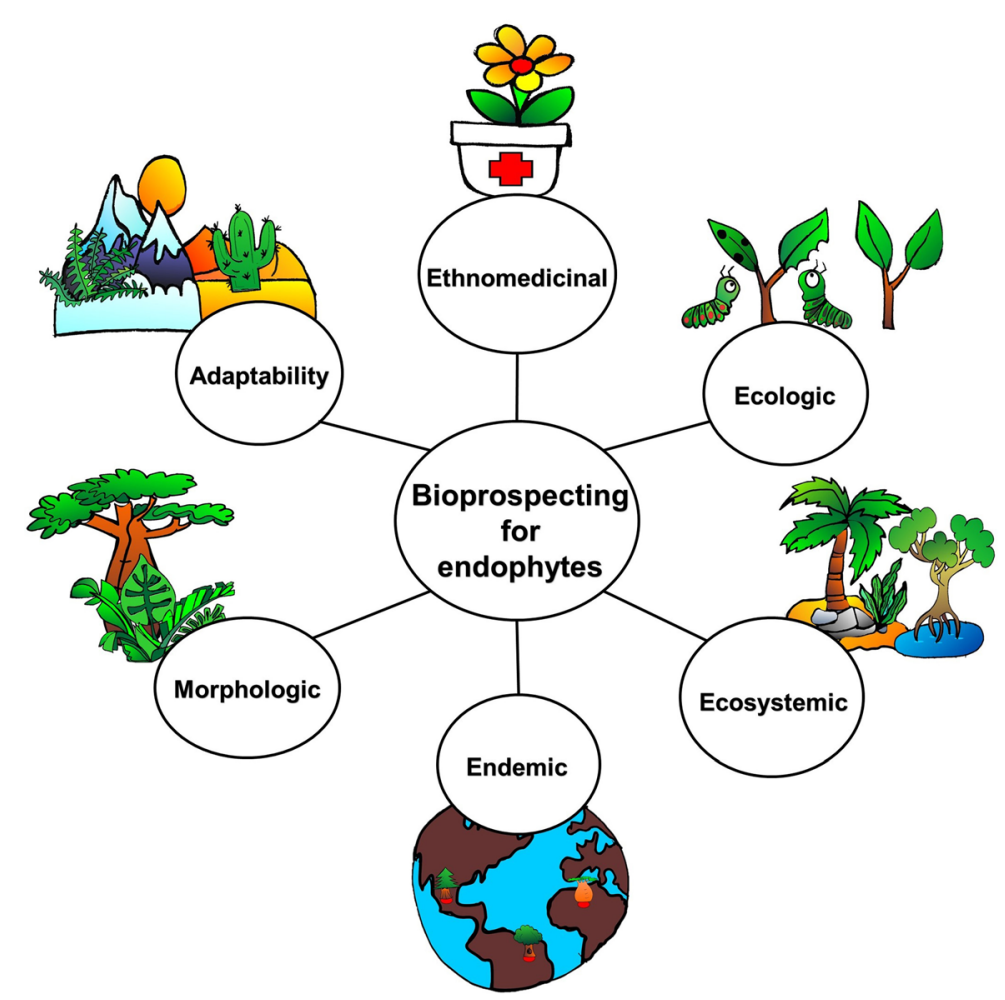

Figure 2

rationale, selecting plants that live in extreme or unique environments, like in the desert, near volcanoes, or in extremely cold weather. These plants might contain endophytes that help them resist or adapt to those harsh conditions. Using the ecosystemic rationale, scientists select plants that live in highly biodiverse ecosystems. Interestingly, it appears that plants growing in tropical or semitropical areas of the world, where there are many types of plants, have a greater diversity of endophytes than those growing in ecosystems with lower plant diversity [3]. The morphologic rationale guides scientists to select a plant with uncommon physical features. For example, the form of the leaves, the shape of the trunk, or the presence of unique structures. Finally, using the endemic rationale, native plants from a particular region or plants that live a very long time are selected, for example the Mexican "Tule tree" (Taxodium mucronatum) from Oaxaca, or the "Yax-ché" tree (Ceiba petandra) from Yucatán. These plants could contain endophytes that help to slow down the aging process (Figure 2).

\section{HOW CAN WE EXTRACT THE ENDOPHYTES FROM PLANTS?}

There are different steps involved in getting the endophytes out of plants and growing them in the laboratory. In Figure 3, you can see that we selected a plant known in Mexico as "Tulipán moteado" (Hibiscus rosasinensis). We collected some healthy plants and washed the surface 
Figure 3

How do we extract the endophytes from a plant? (A) Select a plant. (B) Disinfect the surfaces of the plant's tissues. (C) Cut up the disinfected tissues. In

this panel you can appreciate the cells of endophytic fungi stained with Aniline blue (dark arrows) living among the plant cells (white arrows). (D) Put the tissues on agar plates so that the endophytes emerge out of the plant's tissues, then isolate the endophytes growing them on other agar plates. (E) The endophytes are seeded in bioreactors, where they can grow in a liquid broth and transform nutrients into other chemicals.

(F) Filter the broth to separate the microbial cells from the broth.

(G) Extract the chemicals from the broth. (H) The chemicals produced by the endophytes are identified using different procedures. Here you cou can observe chemicals known as terpenes (purple bands), which have different biological properties.

\section{BIOREACTORS}

Recipients with nutrients were organisms grow and produce chemicals.

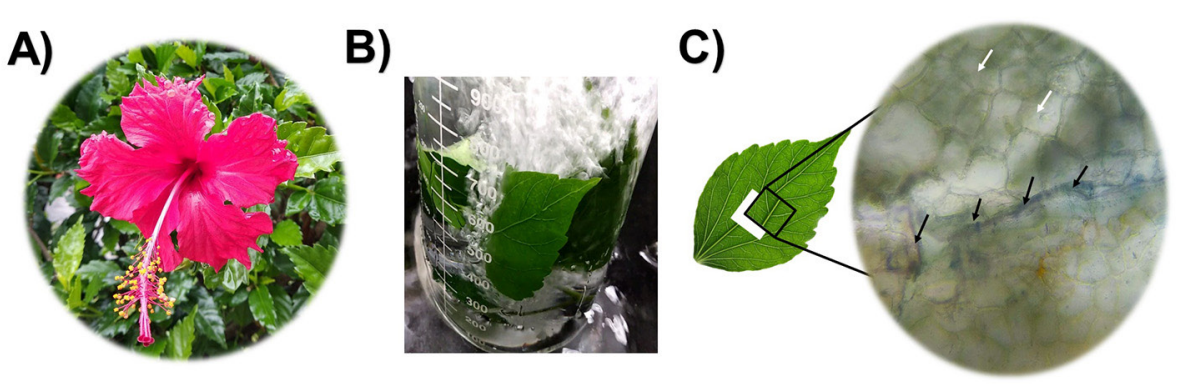

D)

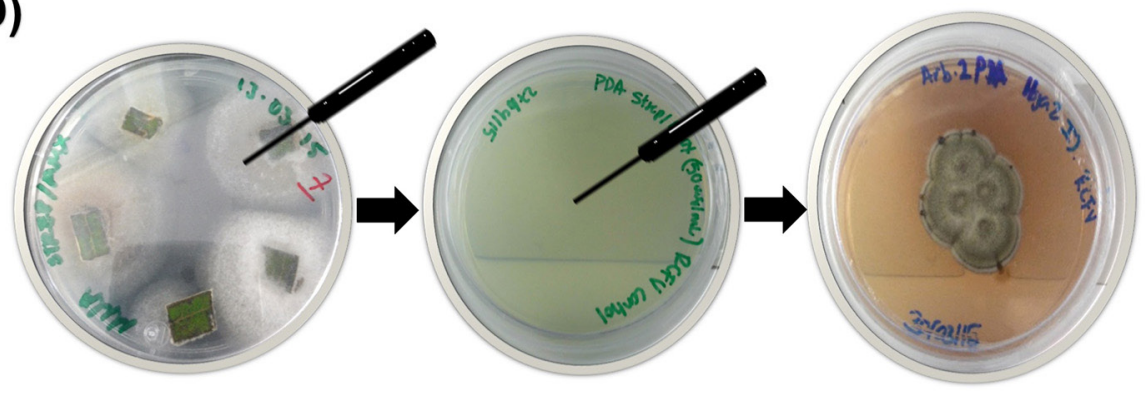

E)

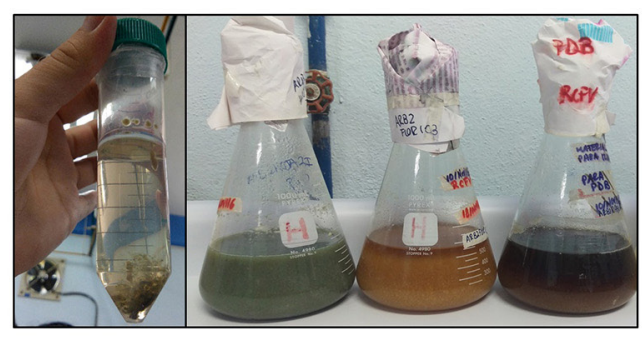

G)

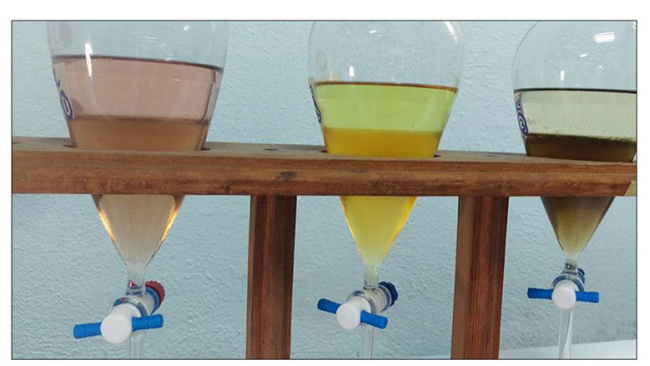

F)

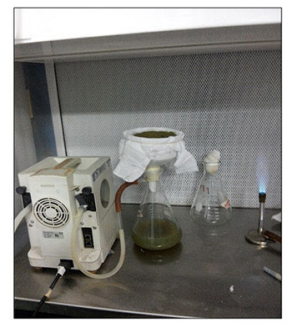

H)

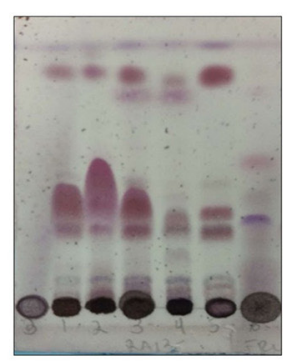

Figure 3

of the plant tissues, like the leaves, with different disinfecting agents. Then, the plant tissues get chopped up, and the pieces are placed inside plates that contain a jelly-like substrate known as agar, which includes nutrients and water for the endophytes to grow and come out of the plant tissues. Reliable disinfection procedures include a final step that confirms that the epiphytes living on the surface were removed from the plant's tissues, so that they do not sneak in the study. Next, the endophytes are removed from the agar plates and grown inside bigger containers full of nutrient broth, also known bioreactors. While in the bioreactors, the endophytes transform the nutrients into other chemicals. Eventually, the endophytes are separated from the broth, and the 
chemicals are extracted from the broth, so that scientists can investigate the identity of the chemicals and their potential uses (Figure 3).

It is important that you know that, up to date, only between 1 and $10 \%$ of the microbes living in an ecosystem can be cultured in the laboratory, leaving 0.9 million fungi and bacteria yet to be identified [3]. If such a great amount of microbes, including the endophytes, cannot be cultured in the laboratory, then how could we study them? For this cases scientists often extract the microbial DNA found inside the plants, then they look for specific pieces of microbial DNA that do not change a lot over time. This conserved pieces of DNA serve as a "barcode" of a particular microbe, this pieces are compared against computational libraries of barcodes that help researchers to identify the majority of unculturable endophytes, and thus have a more integral scope about the biodiversity of microbes that inhabit plants.

\section{ENDOPHYTES FROM PLANTS GROWING IN MEXICO}

Different groups of scientists have discovered endophytes from plants that grow in Mexico, and found that the endophytes can be used to make medicines and produce certain food or cosmetic products. Here are some interesting examples of these discoveries.

The medicinal plant known as "Copalchi" (Hintonia latiflora) contains an endophyte that produces chemicals that could be used to treat diabetes [4]. From other medicinal plant species, like "Granadilla" (Callicarpa acuminata) and the "Copal" tree (Bursera simaruba), scientists found endophytes that produce chemicals that can kill weeds and fight against fungi that cause plant diseases [4]. Endophytes were also found from the medicinal tree "Cuachalalate" (Amphiterygium adstringens), and these endophytes could be used in the production of chemicals to treat cancer or bacterial infections in humans [5]. From the medicinal plant "Mano de oso" (Dendropanax arboreous), scientists discovered endophytes that could produce chemicals to fight against noxious microbes that make humans sick [6].

Endophytes from plants that Mexicans eat in their daily meals were also studied. For example, scientists studied endophytes living in "Tomatillo" (Physallis ixocarpa) [7], which is used to prepare delicious sauces. Scientists that studied the coffee plant (Coffea arabica) growing in Veracruz were interested in investigating whether environmental conditions played a role in the number or type of endophytes living in a plant. And they found that the diversity of endophytes and their communities inside the coffee plant could be owed mainly to the region in which the coffee is grown [8]. And at last but not least, a group of 
scientists identified some endophytes from maize plants, which are used to make "tortillas" [9]. These endophytes can help plants to more easily absorb the nutrients that they need, helping the plants to grown even with a lower amount of fertilizer.

In this article, you got to know about endophytes, the hidden microbial helpers living inside plants. And, as you might guess, there are still plenty of endophytes waiting to be discovered. Are you ready to take the challenge and become an amazing Endophyte Hunter?

\section{ACKNOWLEDGMENTS}

This research was supported by CONACyT through the grants for Basic Science [\#386198] awarded to MLV, and grant for Graduate studies awarded to RCFV [\#222714]. This product is part of M.Sc. thesis of RCFV.

\section{REFERENCES}

1. Zhang, H. W., Song, Y. C., and Tan, R. X. 2006. Biology and chemistry of endophytes. Nat. Prod. Rep. 23:753-71. doi: 10.1039/b609472b

2. Stierle, A., Strobel, G., Stierle, D., Grothaus, P., and Bignami, G. 1995. The search for a taxol-producing microorganism among the endophytic fungi of the Pacific yew, Taxus brevifolia. J. Nat. Prod. 58:1315-24. doi: 10.1139/w02-023.

3. Strobel, G., and Daisy, B. 2003. Bioprospecting for microbial endophytes and their natural products. Microbiol. Mol. Biol. Rev. 67:491-502. doi: 10.1128/ MMBR.67.4.491-502.2003

4. Mata, R., Figueroa, M., Rivero, I., and Macías, M. L. 2018. Insights in fungal bioprospecting in Mexico. Planta Med. 84:594-605. doi: 10.1055/s-0044-101551

5. Centeno-Leija, S., Vinuesa, P., Rodríguez-Peña, K., Trenado-Uribe, M., Cárdenas-Conejo, Y., Serrano-Posada, H., et al. 2016. Draft genome sequence of an endophytic Actinoplanes species, encoding uncommon transacyltransferase polyketide synthases. Genome Announc. 4:e00164-16. doi: 10.1128/genomeA.00164-16

6. Ramos-Garza, J., Rodríguez-Tovar, A. V., Flores-Cotera, L. B., Rivera-Orduña, F. N., Vásquez-Murrieta, M. S., Ponce-Mendoza, A., et al. 2016. Diversity of fungal endophytes from the medicinal plant Dendropanax arboreus in a protected area of Mexico. Ann. Microbiol. 66:991-1002. doi: 10.1007/s13213-015-1184-0

7. Marquez-Santacruz, H. A., Hernandez-Leon, R., Orozco-Mosqueda, M. C., Velazquez-Sepulveda, I., and Santoyo, G. 2010. Diversity of bacterial endophytes in roots of Mexican husk tomato plants (Physalis ixocarpa) and their detection in the rhizosphere. Gen. Mol. Res. 9:2372-80. doi: 10.4238/ vol9-4gmr921 
8. Saucedo-García, A., Anaya, A. L., Espinosa-García, F. J., and González, M. C. 2014. Diversity and communities of foliar endophytic fungi from different agroecosystems of Coffea arabica L. in two regions of Veracruz, Mexico. PLoS ONE 9:e98454. doi: 10.1371/journal.pone.0098454

9. Estrada, P., Mavingui, P., Cournoyer, B., Fontaine, F., Balandreau, J., and Caballero-Mellado, J. 2002. A $\mathrm{N}_{2}$-fixing endophytic Burkholderia sp. associated with maize plants cultivated in Mexico. Can. J. Microbiol. 48: 285-94. doi: 10.1139/w02-023.

SUBMITTED: 02 September 2018; ACCEPTED: 17 January 2019; PUBLISHED ONLINE: 06 February 2019.

EDITED BY: Francisco Barona-Gomez, Centro de Investigación y de Estudios Avanzados del Instituto Politécnico Nacional, Mexico

CITATION: Flores-Vallejo RdC, Folch-Mallol JL, Sharma A, Cardoso-Taketa AT and Villarreal ML (2019) Hidden Microbial Helpers Living Inside Plants: Getting to Know Endophytes and Some of Their Applications in Our Daily Lives. Front. Young Minds 7:11. doi: 10.3389/frym.2019.00011

CONFLICT OF INTEREST STATEMENT: The authors declare that the research was conducted in the absence of any commercial or financial relationships that could be construed as a potential conflict of interest.

COPYRIGHT @) 2019 Flores-Vallejo, Folch-Mallol, Sharma, Cardoso-Taketa and Villarreal. This is an open-access article distributed under the terms of the Creative Commons Attribution License (CC BY). The use, distribution or reproduction in other forums is permitted, provided the original author(s) and the copyright owner(s) are credited and that the original publication in this journal is cited, in accordance with accepted academic practice. No use, distribution or reproduction is permitted which does not comply with these terms.

\section{YOUNG REVIEWERS}

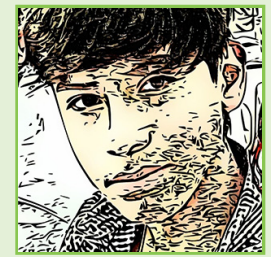

\section{ALAN, AGE: 13}

I am currently in the second year of high school. I am very observant, have a very good memory, and am interested in technology and videogames. I am also interested in maritime animals, and in the bacterial world. I would like to study medicine, because I am struck by the functioning of the human body and I want to help others.

\section{MARIANA, AGE: 14}

I am a student from Guanajuato, México and I am a squash player. I am in the national junior squash team and I am a volunteer for the Lions Club from Guanajuato. My family consists of my mom, my dad, and my two brothers. I have two lovely pets, one of them is named Han Solo and the other one is called Squash. 



\section{AUTHORS}

\section{ROSARIO DEL CARMEN FLORES-VALLEJO}

From a very young age I was eager to ask a lot of questions, and found in the study of Chemistry and Biology very interesting and breath-taking answers. This later encouraged me to study Biotechnology Engineering. Currently I work as a Research Assistant, and continue in the quest for answers exploring the endomicrobiome of plants. I aim to harness the knowledge of plant-microbe molecular interactions to obtain novel natural products that combat of pro-inflammatory diseases and drugresistant bacteria. When I am not at the lab some of my favorite activities are painting, photography, and cycling. *rosario.floresv@uaem.edu.mx

\section{JORGE LUIS FOLCH-MALLOL}

Since I was a child, for the horror of the women in my family, I started small insect zoo at home. I had praying mantis, dung beetles, stick insects, and other bugs, including a small water snake. Obviously, I studied Biology and became a fan of mushrooms (or fungi). These organisms fascinated me specially because they cannot move, so if a lion comes for them, they cannot escape like zebras or antelopes. So, how do they manage to stay alive with so many predators around? Finally, I got my own lab and devoted myself to study several aspects of fungi.

\section{ALEXANDRE CARDOSO-TAKETA}

I am currently a researcher in the Lab of Medicinal Plants at the Autonomous University of the State of Morelos. My research focuses on medicinal plants used in the Mexican traditional medicine, including ferns with neuropharmacological properties. I am also interested in methodologies like NMR, UPLC/GC-MS, and HTPLC for metabolomics analysis of plants. It is fascinating for me to discover new molecules that can improve people's lives. In my free time I like to cook, fusing the flavors of Brazilian and Japanese culture, which I carry in my blood, with the strong flavors of Mexico.

\section{MARIA LUISA VILLARREAL}

My interest in Biology started when I was 6 years old, when my dad showed me the beauty of living organisms in a microscope. I studied biology and became a biotechnologist looking for actual tools that can help to improve the quality of life. I focused in plants, searching for the incredible and important secondary metabolites that they produce to be used for human health. For 25 years, I have conducted a research group at the Autonomous University of the State of Morelos that was able to discover interesting molecules with pharmacological value from Mexican medicinal plants. I love to travel in my free times. *luisav@uaem.mx

\section{ASHUTOSH SHARMA}

I grew up in Latipur, India. I was awarded with a fellowship and obtained my Ph.D. degree in Mexico, were I studied medicinal plants used in the treatment of nervous disorders. I became a scientist and full-time professor, specialized in the fields of: plant biotechnology, ethnobotany, bioinformatics, plant biotechnology, genomics, and metabolomics. Currently I am the regional leader of the Department of Bioengineering, at Tec de Monterrey, Queretaro. I am a fluent speaker of many languages, and on my free time I enjoy fishing and spending time with my family. 